International Journal of Bifurcation and Chaos, Vol. 28, No. 4 (2018) 1830011 [12 pages)

(C) The Author(s)

DOI: 10.1142/S0218127418300112

\title{
Chaotic Itinerancy Observed in Mutually Coupled Gaussian Maps
}

\author{
Mio Kobayashi \\ Department of Creative Technology Engineering, \\ National Institute of Technology, \\ Anan College, 265 Aoki Minobayashi, \\ Anan, Tokushima 774-0017, Japan \\ kobayashi@anan-nct.ac.jp \\ Tetsuya Yoshinaga \\ Graduate School of Biomedical Sciences, \\ Tokushima University, 3-18-15 Kuramoto, \\ Tokushima 770-8509, Japan \\ yosinaga@medsci.tokushima-u.ac.jp
}

Received February 5, 2018

\begin{abstract}
A one-dimensional Gaussian map defined by a Gaussian function describes a discrete-time dynamical system. Chaotic behavior can be observed in both Gaussian and logistic maps. This study analyzes the bifurcation structure corresponding to the fixed and periodic points of a coupled system comprising two Gaussian maps. The bifurcation structure of a mutually coupled Gaussian map is more complex than that of a mutually coupled logistic map. In a coupled Gaussian map, it was confirmed that after a stable fixed point or stable periodic points became unstable through the bifurcation, the points were able to recover their stability while the system parameters were changing. Moreover, we investigated a parameter region in which symmetric and asymmetric stable fixed points coexisted. Asymmetric unstable fixed point was generated by the $D$-type branching of a symmetric stable fixed point. The stability of the unstable fixed point could be recovered through period-doubling and tangent bifurcations. Furthermore, a homoclinic structure related to the occurrence of chaotic behavior and invariant closed curves caused by two-periodic points was observed. The mutually coupled Gaussian map was merely a two-dimensional dynamical system; however, chaotic itinerancy, known to be a characteristic property associated with high-dimensional dynamical systems, was observed. The bifurcation structure of the mutually coupled Gaussian map clearly elucidates the mechanism of chaotic itinerancy generation in the two-dimensional coupled map. We discussed this mechanism by comparing the bifurcation structures of the Gaussian and logistic maps.
\end{abstract}

Keywords: Gaussian map; logistic map; bifurcation phenomena; chaos; chaotic itinerancy.

\footnotetext{
This is an Open Access article published by World Scientific Publishing Company. It is distributed under the terms of the Creative Commons Attribution 4.0 (CC-BY) License. Further distribution of this work is permitted, provided the original work is properly cited.
} 


\section{Introduction}

Various nonlinear phenomena, including periodic and nonperiodic oscillations as well as chaotic behavior, have intrigued multiple researchers in many disciplines, such as biology, chemistry, physics, neurobiology, and engineering Gandomi et al., 2013; Cho et al., 1987; Berger et al., 2007; Gilad et al., 2004]. Nonlinear phenomena have been studied through the analysis of numerical models based on dynamical systems and bifurcation theory Baneriee \& Grebogi, 1999; Wieczorek et al., 2002; Orosz et al., 2004; Balanov et al., 2005]. Differential equations and iterated maps are the two main types of numerical and complex systems that have been investigated Guckenheimer \& Holmes, 2013]. Since differential equations can describe the evolution of systems in continuous time, the numerical integration of differential equations could well represent the complicated behaviors of experimental subjects. However, numerical integration tends to accumulate numerical errors over time and can be extremely time-consuming. The study and analysis of iterated maps, e.g. discrete-time dynamical systems described by difference equations, are considerably important because the complicated properties associated with dynamical systems could be reproduced by simple models, using which these properties could be clearly observed. In addition, describing models in simple forms is necessary when we consider the applications of such models Aftab \& Shafia, 2015; Han et al., 2017; Gámez et al., 2017]. The simplicity of numerical models is the key to improving the comprehensibility of the mechanism of the phenomena observed in the models and implementability of the applications.

For a simple discrete-time dynamical system, a logistic map is a model that can represent the dynamics of population growth Mav. 1976: Verhulst, 1845]. Even though the model is a one-dimensional dynamical system, a logistic map can generate various phenomena, including periodic points and chaotic behavior. Hence, many researchers have shown interest in logistic maps and one-dimensional models Tarasova \& Tarasov. 2017: Lampart \& Oprocha, 2016; Maranhad, 2016].

In contrast, Gaussian maps, which are also one-dimensional maps based on the Gaussian exponential function [Hilborn, 2000], have drawn less attention because of their similarity to logistic maps. A Gaussian map also generates various phenomena including periodic points and chaotic behavior, when the system parameters are varied. Since the function of a Gaussian map is bell-shaped, which is similar to the shape of a logistic map, the dynamics of a Gaussian map seems to have properties similar to those of a logistic map. However, there has not yet been a sufficient discussion on the difference between the dynamics of the Gaussian and logistic maps. Since a logistic map can be considered as an expression with zero-, first-, and second-order terms of a Taylor-expanded Gaussian map around zero, we can discuss the function and influence of higher-order terms, which are omitted in approximate systems, by investigating the difference between the Gaussian and logistic maps. In particular, the appearance of coexisting attractors in a Gaussian map Patidar \& Sud, 2009; Patidar, 2006] is an interesting subject of investigation as they do not appear in a logistic map.

Herein, to elucidate the difference between the Gaussian and logistic maps, mutually coupled Gaussian and logistic maps are investigated in terms of the bifurcation phenomena. Focusing on the differences in the properties of the maps, we investigated the bifurcation structure of each coupled map. Based on bifurcation analysis, the chaotic behaviors observed in a mutually coupled Gaussian map and logistic map are described. Furthermore, in a mutually coupled Gaussian map, we observed chaotic itinerancy Kaneko \& Tsuda, 2003; Tsuda, 2015 in specific chaotic behavior. Generally, chaotic itinerancy has been thought of as characteristic phenomena generated in high-dimensional dynamical systems Kaneko, 1991; Ikeda et al., 1989; Tsuda \& Umemura, 2003]. The basic characteristics of chaotic itinerancy include existence of low-dimensional ordered motion which is called attractor-ruin Kaneko \& Tsuda, 2003]. In our proposed model which is a two-dimensional coupled map, we observed attractor-ruins and transition motion between those attractor-ruins. Even though transition of chaotic attractors between low- and high-dimensional motion did not appear in our proposed model, the two-dimensional coupled map is a minimum structure which generates chaotic itinerancy. In addition, based on the bifurcation analysis, we investigated the mechanism of the generation of chaotic itinerancy in a mutually coupled Gaussian map.

Herein, we first address the results of the bifurcation analysis focusing on a fixed point and twoperiodic points observed in each mutually coupled 
map and then highlight the difference between the bifurcation structures of the coupled Gaussian and logistic maps. Then, the chaotic itinerancy observed in the mutually coupled Gaussian map is demonstrated and the mechanism that generates chaotic itinerancy is explained based on the bifurcation analysis.

\section{Mutually Coupled Systems of One-Dimensional Maps}

The dynamics of the mutually coupled systems of one-dimensional Gaussian maps are generally described as a difference equation, which can be expressed as follows:

$$
\boldsymbol{x}(t+1)=\boldsymbol{f}(\boldsymbol{x}(t)) .
$$

Equivalently, they can be described as an iterated map, which can be expressed as follows:

$$
\boldsymbol{f}: \mathbb{R}^{2} \rightarrow \mathbb{R}^{2} ; \quad \boldsymbol{x} \mapsto \boldsymbol{f}(\boldsymbol{x}),
$$

where $t$ denotes discrete time, $\mathbb{R}$ represents a set of real numbers, and $\boldsymbol{x}$ and $\boldsymbol{f}$ represent $\left(x_{1}, x_{2}\right)^{\top}$ and $\left(f_{1}, f_{2}\right)^{\top}$, respectively. The dynamics of the mutually coupled systems of the Gaussian and logistic maps discussed herein are described as

$$
\left(\begin{array}{l}
f_{1}(\boldsymbol{x}) \\
f_{2}(\boldsymbol{x})
\end{array}\right)=\left(\begin{array}{l}
\exp \left(-\alpha x_{1}^{2}\right)+\beta+\varepsilon\left(x_{2}-x_{1}\right) \\
\exp \left(-\alpha x_{2}^{2}\right)+\beta+\varepsilon\left(x_{1}-x_{2}\right)
\end{array}\right)
$$

and

$$
\left(\begin{array}{l}
f_{1}(\boldsymbol{x}) \\
f_{2}(\boldsymbol{x})
\end{array}\right)=\left(\begin{array}{c}
\gamma x_{1}\left(1-x_{1}\right)+\beta+\varepsilon\left(x_{2}-x_{1}\right) \\
\gamma x_{2}\left(1-x_{2}\right)+\beta+\varepsilon\left(x_{1}-x_{2}\right)
\end{array}\right),
$$

respectively. $\alpha, \gamma, \beta$, and $\varepsilon$ are system parameters. $\varepsilon$ represents the coupling strength between two connected maps. The systems in Eqs. (3) and (4) possess reflection symmetricity, namely,

$$
P:=\left(\begin{array}{ll}
0 & 1 \\
1 & 0
\end{array}\right)
$$

and

$$
\boldsymbol{f}(P \boldsymbol{x})=P \boldsymbol{f}(\boldsymbol{x})
$$

are satisfied by both mutually coupled Gaussian and logistic maps. Herein, with the settings $\alpha=6$ and $\gamma=2$, we analyze the bifurcation sets of the mutually coupled systems of the Gaussian and logistic maps as the bifurcation parameters by changing the values of $\beta$ and $\varepsilon$.

\section{Method}

In bifurcation analysis, we used a method based on the qualitative bifurcation theory Kawakami, 1984]. The point $\boldsymbol{x}^{*}$ satisfying

$$
\boldsymbol{x}^{*}-\boldsymbol{f}\left(\boldsymbol{x}^{*}\right)=\mathbf{0}
$$

becomes a fixed point in Eq. (3) or Eq. (4). The characteristic equation for the fixed point $\boldsymbol{x}^{*}$ is defined as

$$
\chi\left(\boldsymbol{x}^{*}, \boldsymbol{\mu}\right)=\operatorname{det}\left(\boldsymbol{\mu} \boldsymbol{I}-D \boldsymbol{f}\left(\boldsymbol{x}^{*}\right)\right)=0,
$$

where $\boldsymbol{I}$ is the $2 \times 2$ identity matrix and $D \boldsymbol{f}$ denotes the derivative of $\boldsymbol{f}$. We consider $\boldsymbol{x}^{*}$ to be hyperbolic if none of the absolute eigenvalues of $D \boldsymbol{f}$ are at unity. Note that in Eq. (17), an $m$-periodic point can be investigated by replacing $\boldsymbol{f}$ with $\boldsymbol{f}^{m}$, i.e. the $m$ th iteration of $\boldsymbol{f}$. In the following discussion, we only consider the properties of a fixed point of $\boldsymbol{f}$, though a similar argument can be applied to a periodic point of $\boldsymbol{f}$.

Let us consider the topological classification of a hyperbolic fixed point $\boldsymbol{x}^{*}$. The topological type of a hyperbolic fixed point is determined by $\operatorname{dim} \boldsymbol{E}^{u}$ and $\operatorname{det} \boldsymbol{L}^{u}$, where $\boldsymbol{E}^{u}$ is the intersection of $\mathbb{R}^{2}$ and the direct sum of the generalized eigenspaces of $D \boldsymbol{f}\left(\boldsymbol{x}^{*}\right)$ corresponding to eigenvalue $\boldsymbol{\mu}$ such that $\left|\mu_{i}\right|>1$, and $\boldsymbol{L}^{u}=D \boldsymbol{f}\left(\boldsymbol{x}^{*}\right) \mid \boldsymbol{E}^{u}$.

When $\operatorname{det} \boldsymbol{L}^{u}>0$ and $\operatorname{det} \boldsymbol{L}^{u}<0$, the hyperbolic fixed point is called $D$-type and $I$ type, respectively. Based on this definition, we have five topologically different types of hyperbolic fixed points: ${ }_{k} D, k=0,1,2$ and ${ }_{k} I, k=1,2$. When we consider the distribution of the characteristic multipliers of Eq. (8), $D$ and $I$ correspond to the even and odd numbers, respectively, of the characteristic multipliers on the real axis $(-\infty,-1)$ and $k$ represents the number of the characteristic multipliers outside the unit circle on the complex plane. When all characteristic multipliers are in the unit circle, the topological type is ${ }_{0} D$ that means completely stable; otherwise, ${ }_{k} D, k>0$ and ${ }_{k} I, k>0$ represent directly unstable and inversely unstable, respectively.

Bifurcation occurs when the topological type of a fixed point is changed by the varying of a system parameter. The generic codimension-one bifurcations are the tangent, period-doubling, and Neimark-Sacker bifurcations. In addition, a $D$ type branching appears in a system that possesses some symmetric properties as a degenerate case of the tangent bifurcation. These bifurcations 
are observed when hyperbolicity is destroyed. This corresponds to the critical distribution of the characteristic multiplier $\mu$ such that $\mu=+1$ for tangent bifurcation and $D$-type branching, $\mu=-1$ for period-doubling bifurcation, and $\mu=e^{j \theta}$ for the Neimark-Sacker bifurcation, where $j=\sqrt{-1}$.

The bifurcation sets of a fixed point were computed by solving the simultaneous Eqs. (7) and (8). For numerical determination Kawakami, 1984], we used Newton-Raphson method. The Jacobian matrix of the set of equations was derived from the first and second derivatives of map $f$.

\section{Results}

Using bifurcation analysis, we investigated the bifurcation sets of the mutually coupled Gaussian and logistic maps on the $(\varepsilon, \beta)$-plane. In the bifurcation diagrams, we use the following symbols:

$G_{l}^{m}$ : tangent bifurcation of the $m$-periodic point $I_{l}^{m}$ : period-doubling bifurcation of the $m$-periodic point

$D_{l}^{m}: D$-type branching of the $m$-periodic point $N_{l}^{m}$ : the Neimark-Sacker bifurcation of $m$-periodic point

where $l$ distinguishes the same types of bifurcation sets of $m$-periodic points. As for the fixed points and periodic points observed in the coupled system, $x_{1}=x_{2}$ indicates a symmetrical fixed point or inphase periodic points, whereas $x_{1} \neq x_{2}$ represents an asymmetric fixed point or out-of-phase periodic points.

\subsection{Mutually coupled Gaussian map}

Figure 1 shows the waveforms of the fixed point and two-periodic points at $\varepsilon=-0.2$. As shown in Figs. 1(a) and 1(b), the symmetric and asymmetric periodic points coexist at $\beta=-1.2$. Figures 1(c) and 1(d) show typical in-phase and out-of-phase two-periodic points at $\beta=-0.8$ and $\beta=-0.6$, respectively. Figure 2 shows the two-parameter bifurcation diagram of the fixed point and twoperiodic points observed in the mutually coupled Gaussian map. In Fig. 2, the symmetric stable fixed point exists in the regions represented by

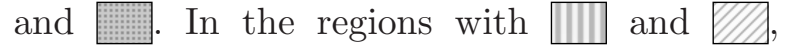
the in-phase stable two-periodic points and out-ofphase stable two-periodic points exist, respectively.
The values of the symmetric stable fixed points existing in the regions $\square$ and $\square$ are different. To explain the parameter region where the fixed point exists, the return map of the single Gaussian map, i.e. the mutually coupled Gaussian map without coupling, is shown in Fig. 3. The intersection of $x(t+1)=x(t)$ and $x(t+1)=\exp \left(-\alpha x^{2}(t)\right)$ corresponds to the fixed point on the line of $\varepsilon=0$, as shown in Fig. 2. In Fig. 3, $x_{s p(\cdot)}$ and $x_{s n(\cdot)}$ represent the stable fixed point at $\beta=-1.2,-0.9$, and 0.42 . In the parameter region where $\beta$ is greater than $G_{1}^{1}$, the fixed point corresponding to $x_{s p(\cdot)}$ always exists even though the stability changes with the varying of the value of $\beta$. When $\beta$ decreases and it passes through $G_{1}^{1}$, the fixed point corresponding to $x_{s p(\cdot)}$ disappears. In contrast, the stable fixed point corresponding to $x_{s n(\cdot)}$ disappears by passing through $G_{2}^{1}$ when $\beta$ increases. The fixed point always exists when $\beta$ is smaller than $G_{2}^{1}$.

Figure 4 shows the stability change of the fixed point and two-periodic points observed in the mutually coupled Gaussian map. The bold solid curves indicate the stable fixed and two-periodic points, while the thin solid curves represent the correspond-

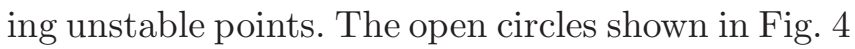
correspond to the bifurcation points. In Fig. 4 , when $\beta$ has a value between $D^{1}$ and $I_{1}^{1}$, two stable fixed points coexist. One of the stable fixed points, the value of which is around zero, becomes unstable when $\beta$ increases and passes through $I_{1}^{1}$, while the stable two-periodic points are generated around the unstable fixed point. After $\beta$ increases, the unstable fixed point passes through $I_{4}^{1}$, and becomes stable again. When we decrease the value of $\beta, D$ type branching appears and the asymmetric unstable fixed point is generated. This point becomes stable after passing through the period-doubling and tangent bifurcations, and at around $\beta=-1.2$, the asymmetric and symmetric stable fixed points coexist.

The generation of the asymmetric fixed point is important for understanding the appearance of chaotic itinerancy, which is comprehensively explained later.

A schematic showing the details of the stability change of the fixed points and the generation of the asymmetric fixed point is illustrated in Fig. 5 . The filled circles $\bigcirc$ and $\bigcirc$ represent tangent bifurcation, D-type branching, and perioddoubling bifurcation, respectively. The solid and 
Chaotic Itinerancy Observed in Mutually Coupled Gaussian Maps
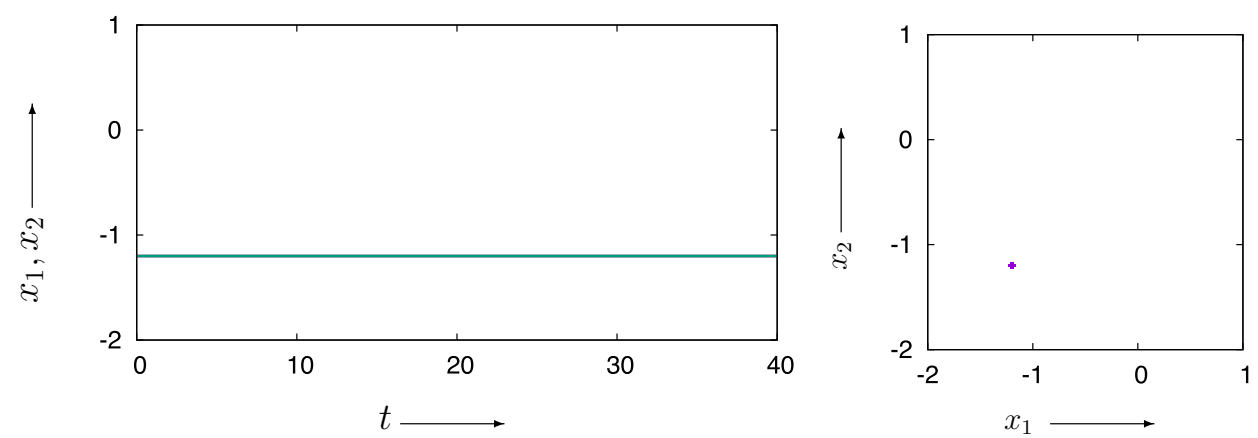

(a)
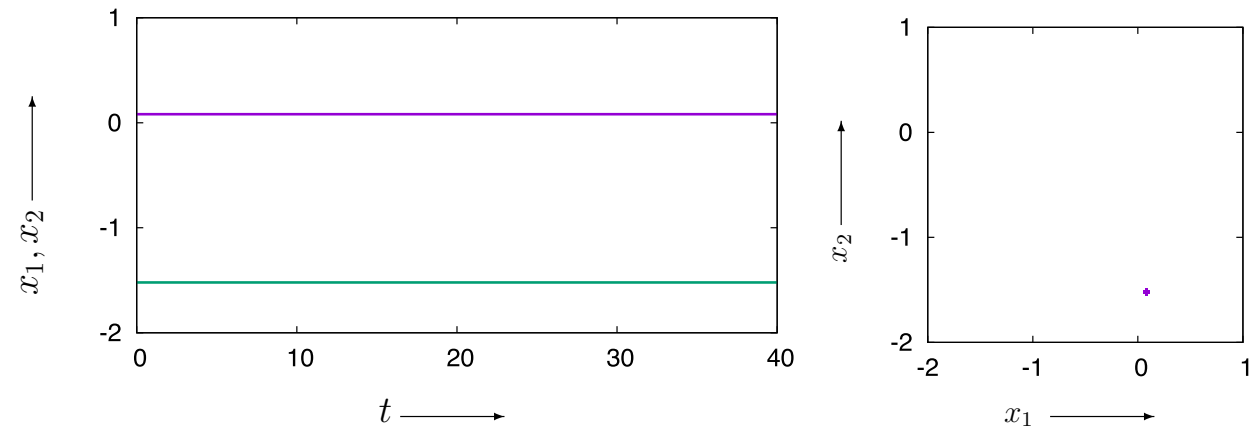

(b)
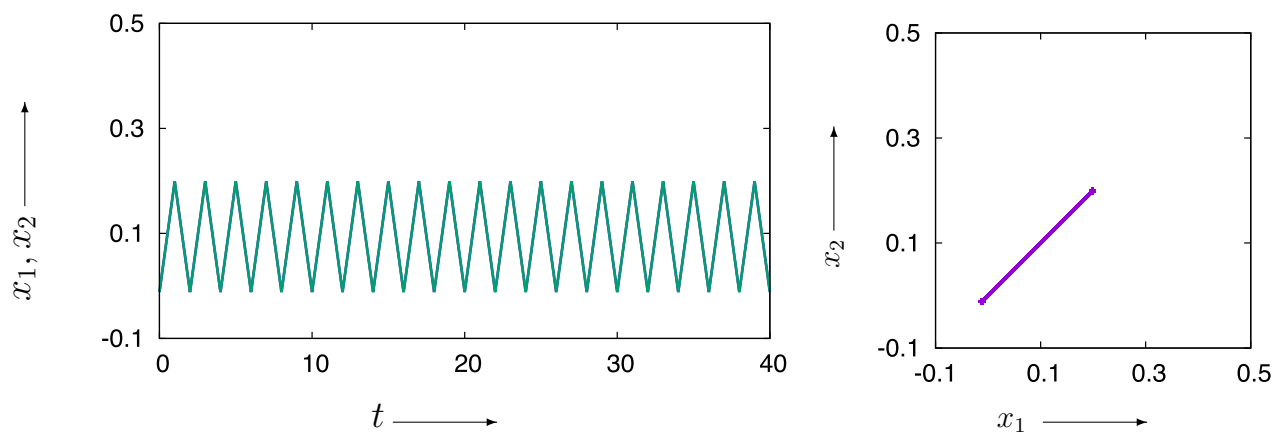

(c)
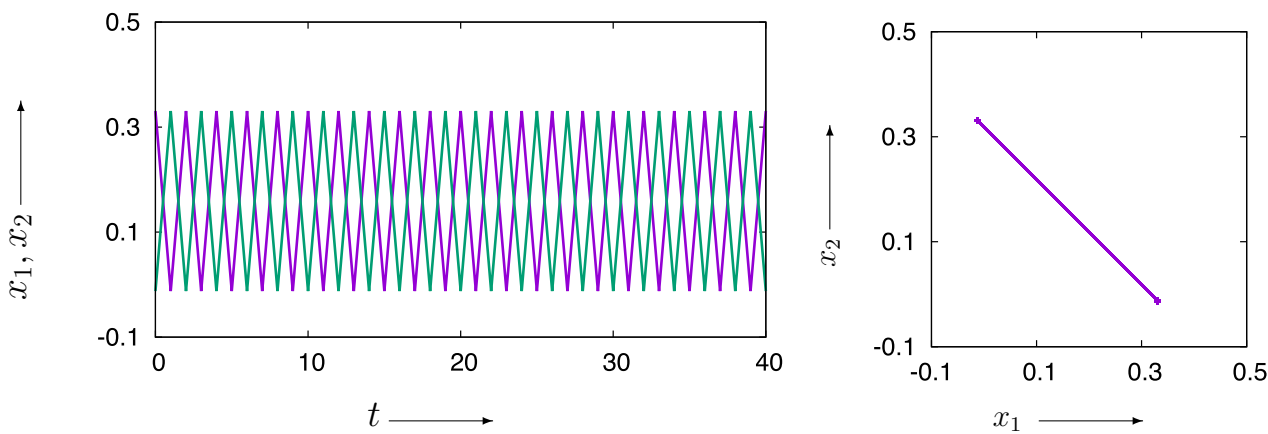

(d)

Fig. 1. Waveforms and phase portraits of a fixed point and two-periodic points at $\varepsilon=-0.2$ and $\alpha=6$. (a) Symmetric fixed point at $\beta=-1.2$, (b) asymmetric fixed point at $\beta=-1.2$, (c) in-phase two-periodic points at $\beta=-0.8$ and (d) out-of-phase two-periodic points at $\beta=-0.6$. 


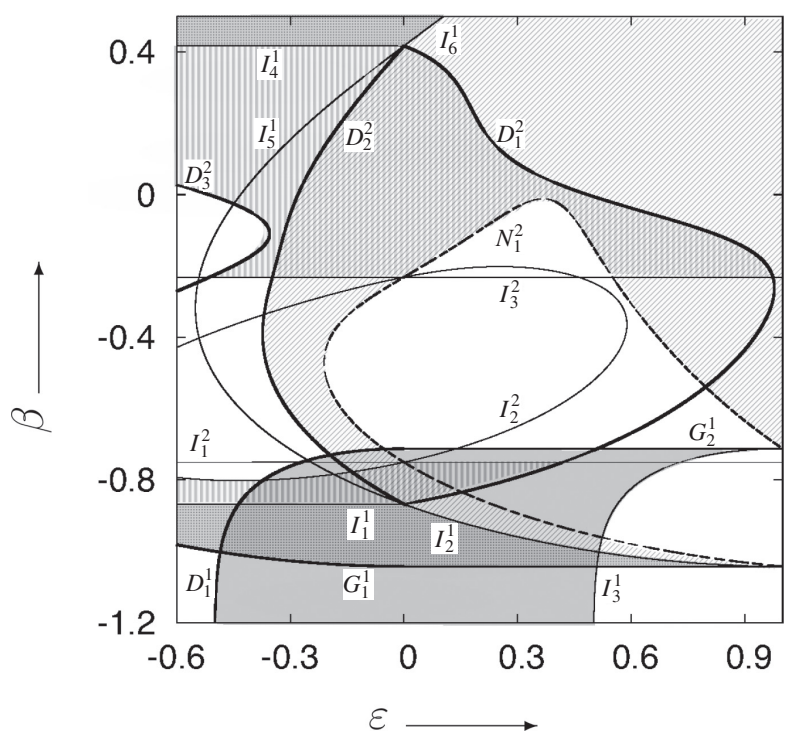

Fig. 2. Bifurcation diagram of a fixed point and twoperiodic points observed in the mutually coupled Gaussian map at $\alpha=6$.

dashed curves indicate the stable and unstable fixed points in Fig. 5. The asymmetric stable fixed point and its period-doubling bifurcation are important for the generation of chaotic itinerancy in the mutually coupled Gaussian map.

Next, we focus on the topological properties of the stable two-periodic points. The in-phase stable two-periodic points exist in the parameter region of $I_{1}^{1}-I_{2}^{2}$ and $I_{3}^{2}-I_{4}^{1}$, whereas the out-of-phase stable two-periodic points exist in the parameter region

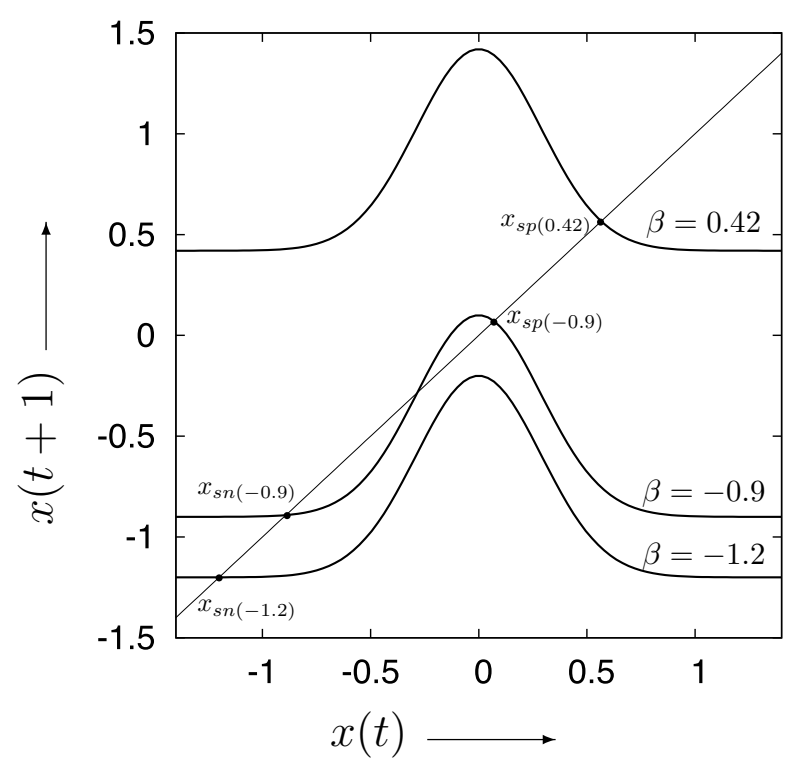

Fig. 3. Return map of Gaussian map at $\alpha=6$.

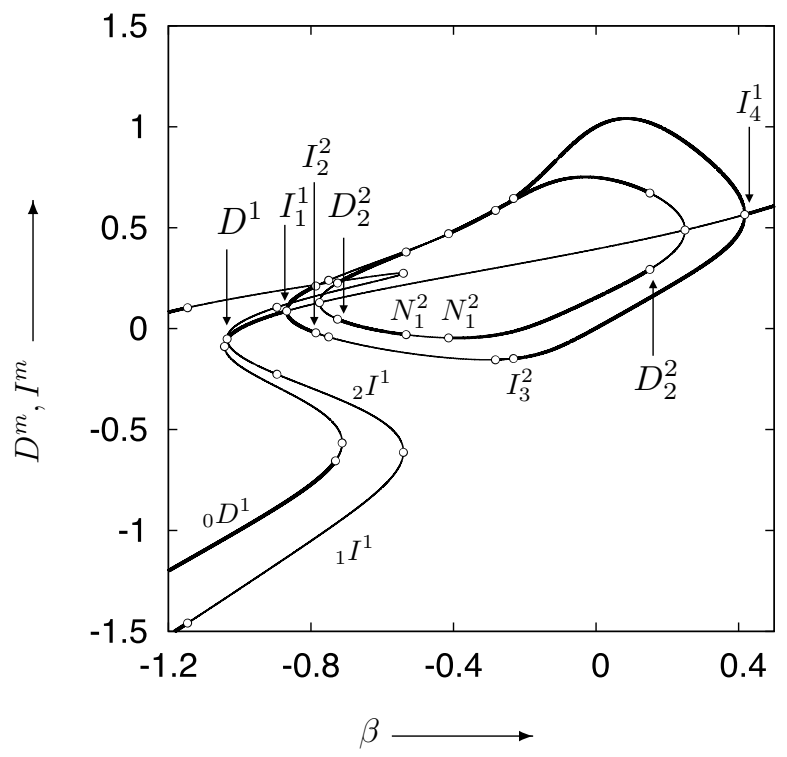

Fig. 4. Stability changes of a fixed point and two-periodic points observed in the mutually coupled Gaussian map at $\varepsilon=-0.2$.

of $D_{2}^{2}-N_{1}^{2}\left(N_{1}^{2}-D_{2}^{2}\right)$. The out-of-phase points are generated by the destruction of the symmetry of the in-phase points. When the two-periodic points pass through $N_{1}^{2}$, an invariant closed curve appears around the out-of-phase stable two-periodic points as $\beta$ is varied. Figure 6 shows the invariant closed curve observed at $\varepsilon=-0.4$ and $\beta=-0.91$. To focus on $x_{1}-x_{2}=0$ or $u=v$, the coordinates are converted to $u=\left(x_{1}+x_{2}\right) / \sqrt{2}$ and $v=\left(x_{1}-x_{2}\right) / \sqrt{2}$. By varying the value of $\beta$, we

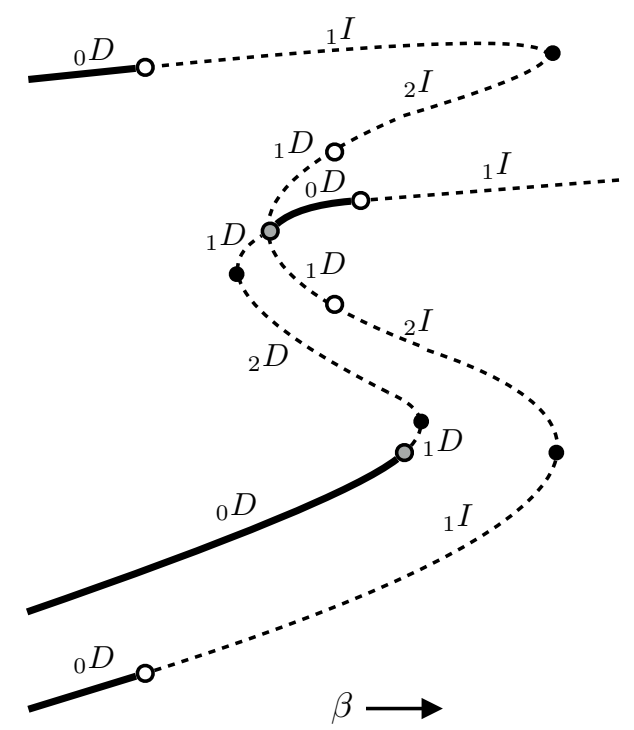

Fig. 5. Schematic diagram on the generation of asymmetric fixed point. 


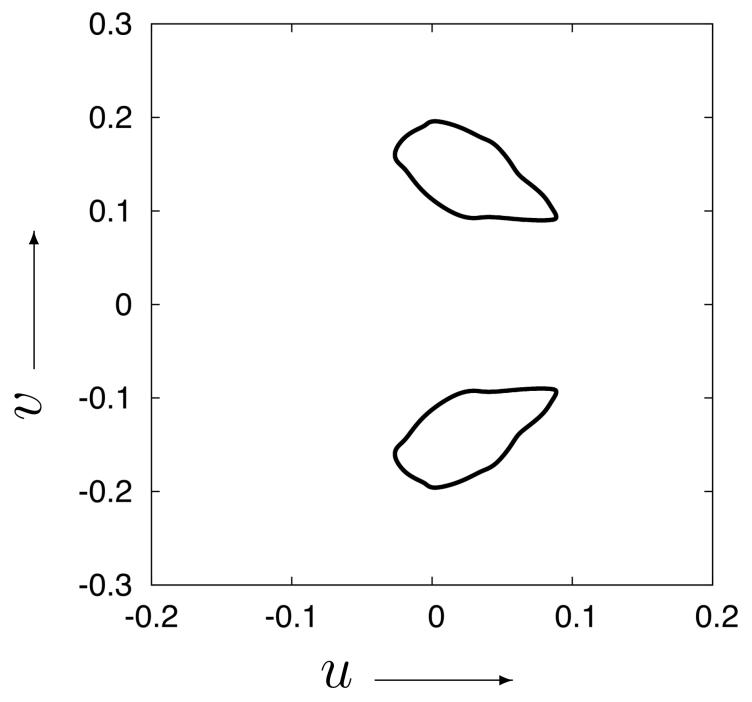

Fig. 6. Invariant closed curve generated by the NeimarkSacker bifurcation of the two-periodic points at $\varepsilon=0.4$ and $\beta=-0.91$.

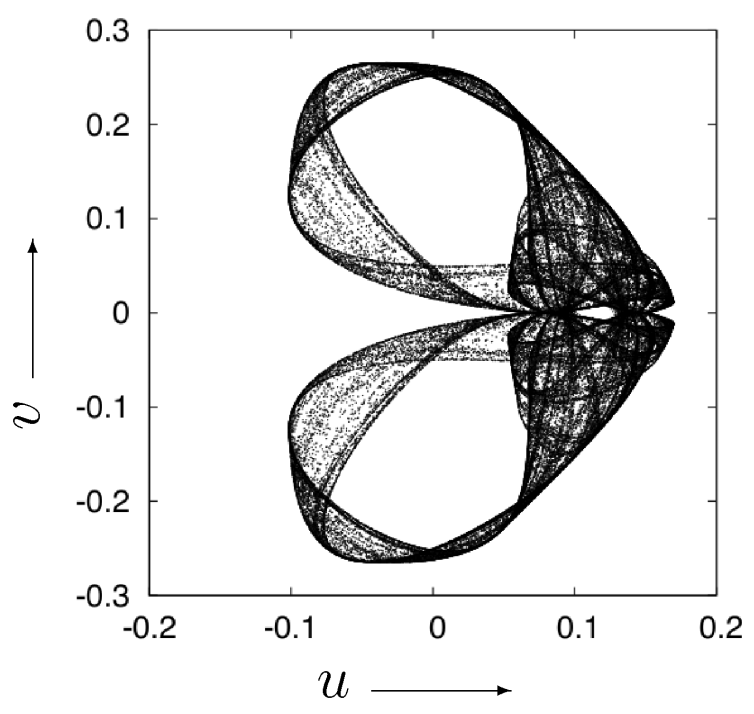

Fig. 7. Chaotic behavior observed at $\varepsilon=0.4$ and $\beta=$ -0.879 . observe the appearance of the transverse and nontransverse (or tangent) types of homoclinic points as well as chaotic behavior, as shown in Fig. 7, Figure 8 shows a waveform corresponding to Fig. 7 at $\varepsilon=0.4$ and $\beta=-0.879$.

\subsection{Mutually coupled logistic map}

Figure 9 shows a two-parameter bifurcation diagram of the mutually coupled logistic map on the $(\varepsilon, \beta)$-plane at $\alpha=6$. In the regions with $\square$, IIII, and ZA, a stable fixed point, in-phase stable periodic points, and out-of-phase stable twoperiodic points exist, respectively. In the parameter region where $\varepsilon$ is greater than $N_{1}^{2}$, an invariant closed curve was observed and chaotic behavior was generated with increasing $\varepsilon$. In the parameter region where $\beta$ is greater than $I_{1}^{1}$, a stable fixed point does not exist. Additionally, when $\beta$ is smaller than $G_{1}^{2}$, a fixed point and periodic points are not observed. This result is understandable since the intersection of $x(t+1)=x(t)$ and $x(t+1)=$ $\gamma x(1-x)$ disappears when $\beta$ decreases, as shown in Fig. 10 ,

Figure 11 represents the change in the stability of the fixed point and two-periodic points at $\beta=$ -0.2 . The bold solid curves indicate the stable fixed point and stable two-periodic points, while the thin solid curves correspond to the unstable fixed point and unstable two-periodic points. In $D^{1}-I_{1}^{1}$, a symmetric stable fixed point exists. By decreasing $\beta$, $D$-type branching appears and the asymmetric and symmetric unstable fixed points are generated. The symmetric unstable fixed point is terminated at $G_{1}^{1}$. On the contrary, by increasing $\beta$, a symmetric stable fixed point passes through $I_{1}^{1}$, after which an unstable fixed point and in-phase stable two-periodic
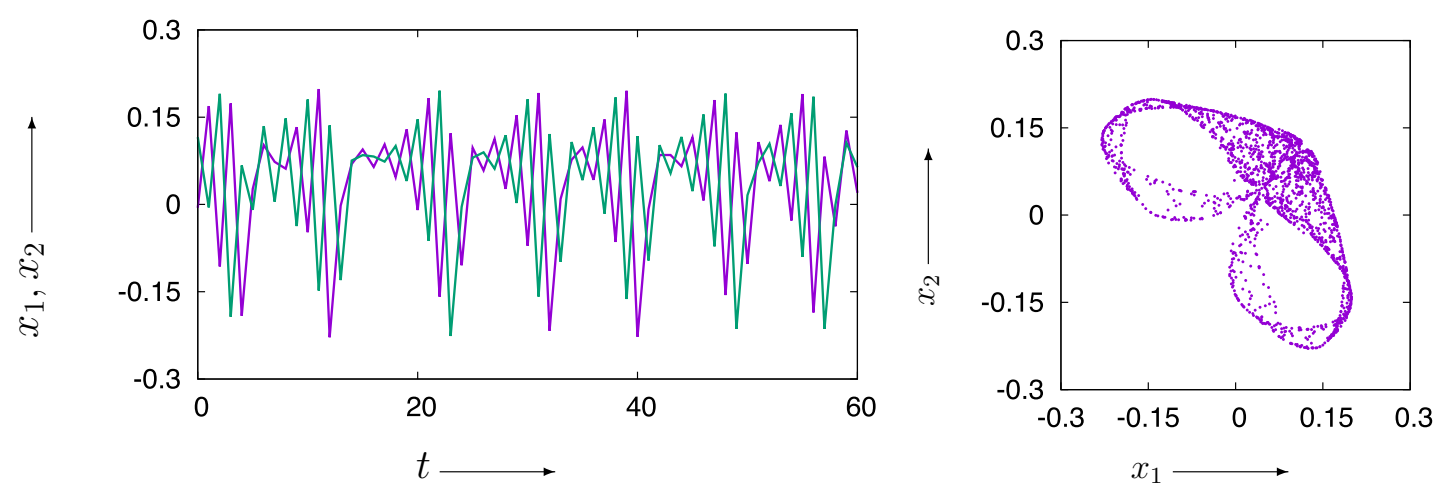

Fig. 8. Waveform and phase portrait at $\varepsilon=0.4, \beta=-0.879$. 


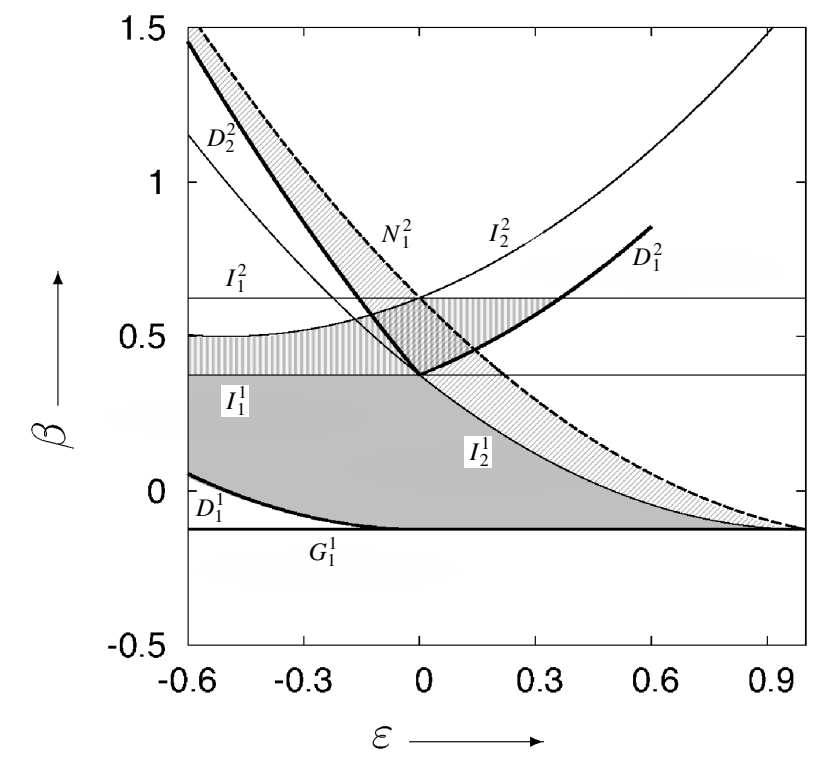

Fig. 9. Bifurcation sets observed in the mutually coupled logistic map at $\gamma=2$.

points are generated. By continuously increasing $\beta$, first, the unstable fixed point passes through $I_{2}^{1}$, after which the in-phase unstable two-periodic points appear. The symmetricity of the in-phase unstable two-periodic points in $I_{2}^{1}-D_{2}^{2}$ is broken via $D$-type branching. Then, the out-of-phase stable two-periodic points are generated in $D_{2}^{2}-N_{1}^{2}$. After passing through $N_{1}^{2}$, an invariant closed curve appears and chaotic behavior is observed by further increasing $\beta$.

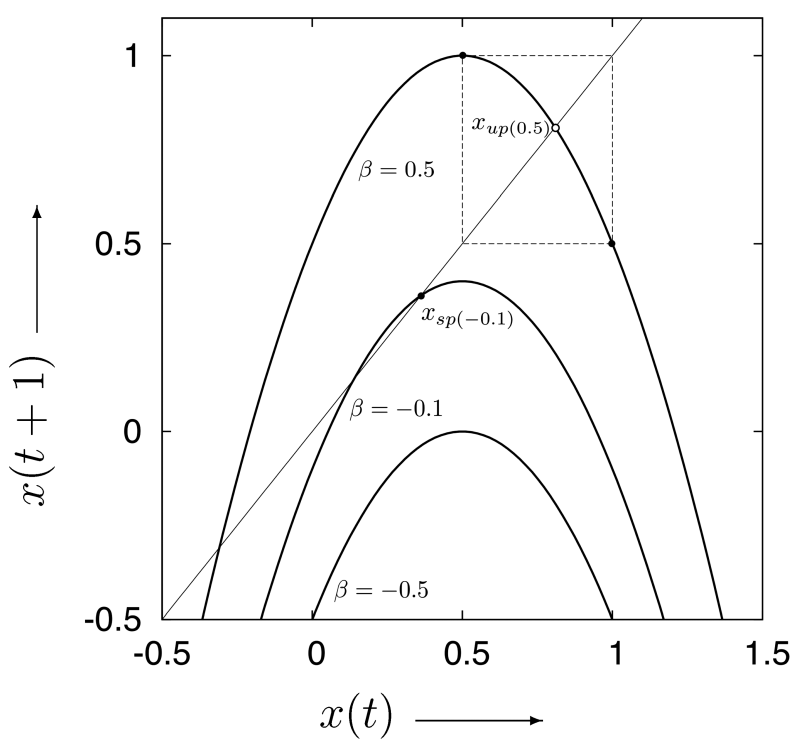

Fig. 10. Return map of the logistic map at $\gamma=2$.

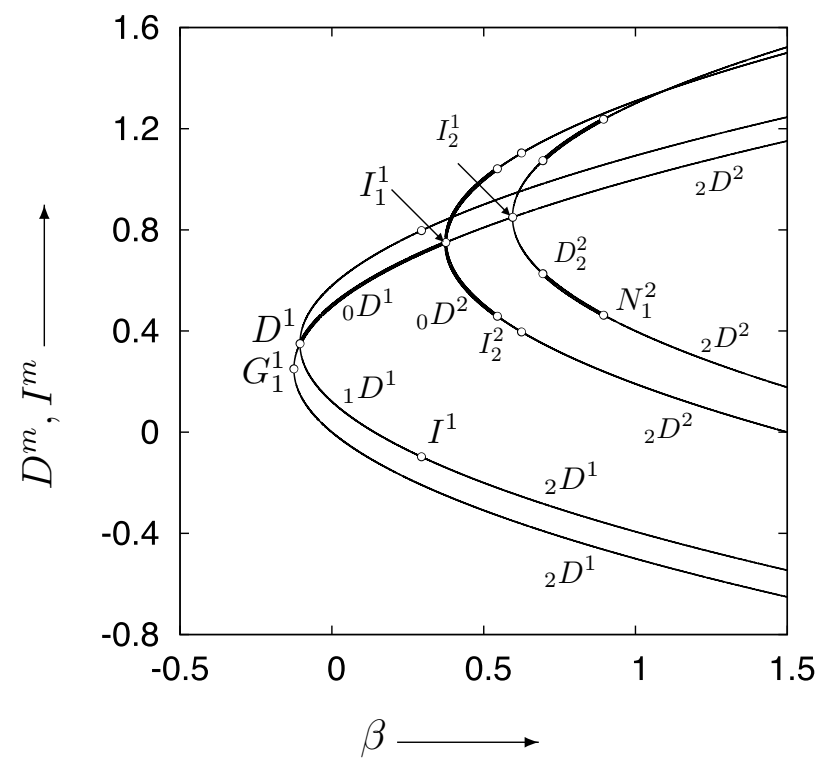

Fig. 11. Stability change of the fixed point and two-periodic points observed in the mutually coupled logistic map at $\varepsilon=-0.2$.

\subsection{Chaotic itinerancy observed in mutually coupled Gaussian maps}

Figure 12 shows a typical waveform of chaotic itinerancy observed in a mutually coupled Gaussian map at $\alpha=12, \beta=-0.504$, and $\varepsilon=-0.086$. After passing enough time to remove a transient state, we plotted the values of $x_{1}$ and $x_{2}$ from $t=0$ to $t=30000$ as shown in Fig. 12(a). Figure 12(b) is an enlarged diagram of Fig. 12(a) between $t=2200$ and $t=3400$. As shown in Fig. 12(b), after one of the two chaotic trajectories is attracted to the other, the trajectories replace each other. Figure 13 indicates the phase portraits of the chaotic itinerancy. The bifurcation structure associated with the asymmetric fixed points, as shown in Fig. 14, explains the mechanism of chaotic itinerancy gen-

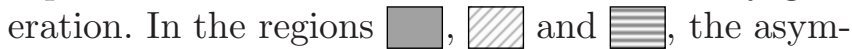
metric stable fixed points, asymmetric in-phase stable two-periodic points, and asymmetric in-phase stable four-periodic points exist, respectively. The right-hand side region $\square$ corresponds to the asymmetric stable fixed point generated via the $D$-type branching of the symmetric stable fixed point, as shown in Fig. 5. The chaotic itinerancy shown in Fig. 12 was observed at the parameter indicated with an X mark in Fig. 14. 


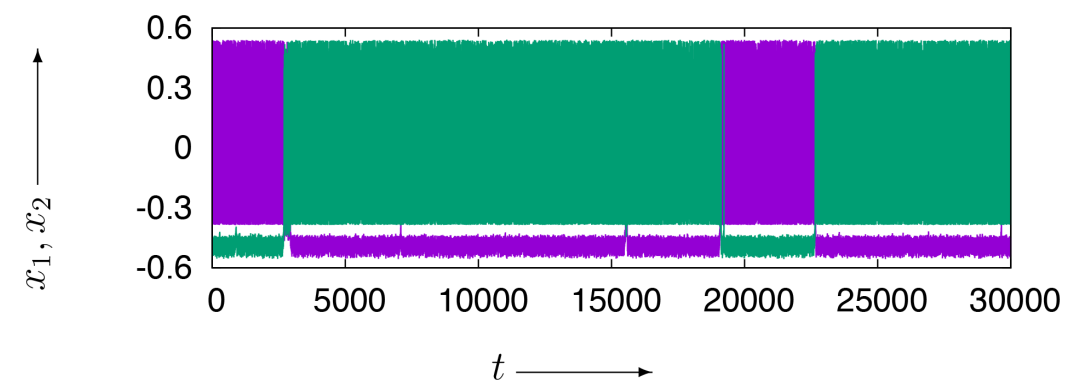

(a)

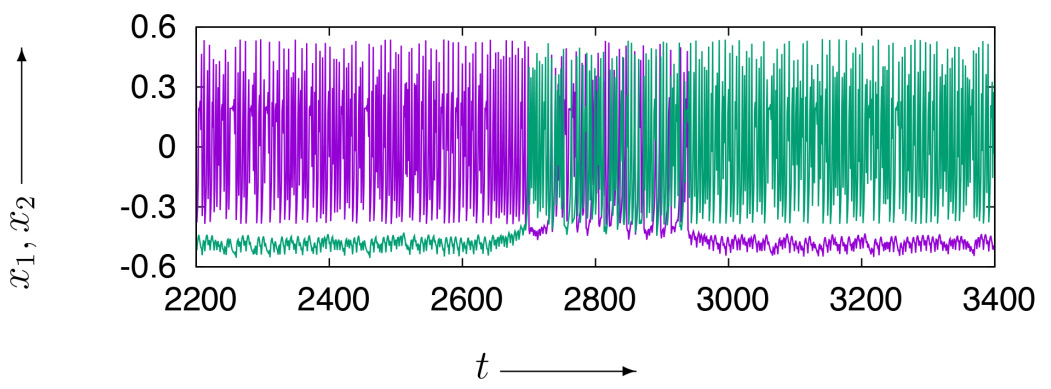

(b)

Fig. 12. Chaotic itinerancy observed in the mutually coupled Gaussian map at $\alpha=12, \beta=-0.504$, and $\varepsilon=-0.086$. (a) Typical waveforms and (b) enlarged diagram of (a).

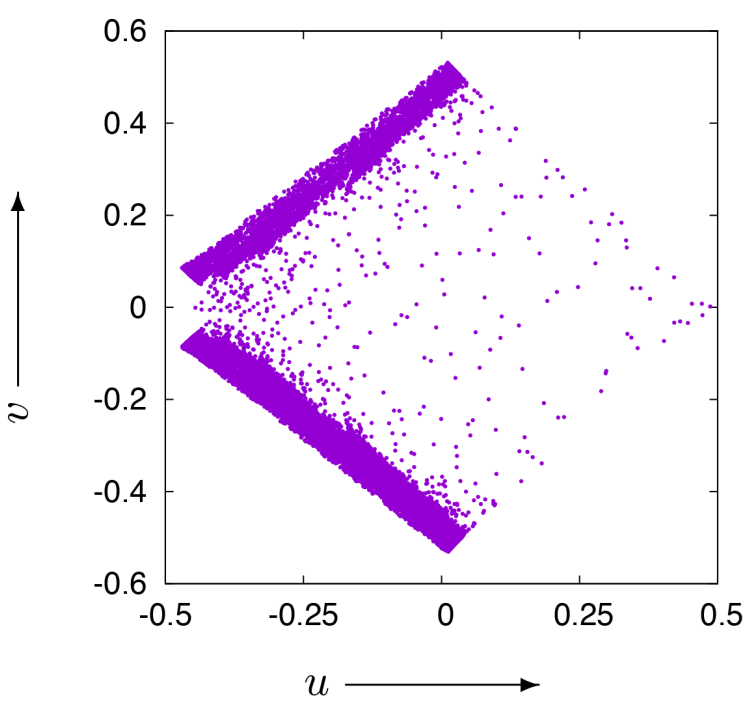

(a) $t=0,1, \ldots, 30000$

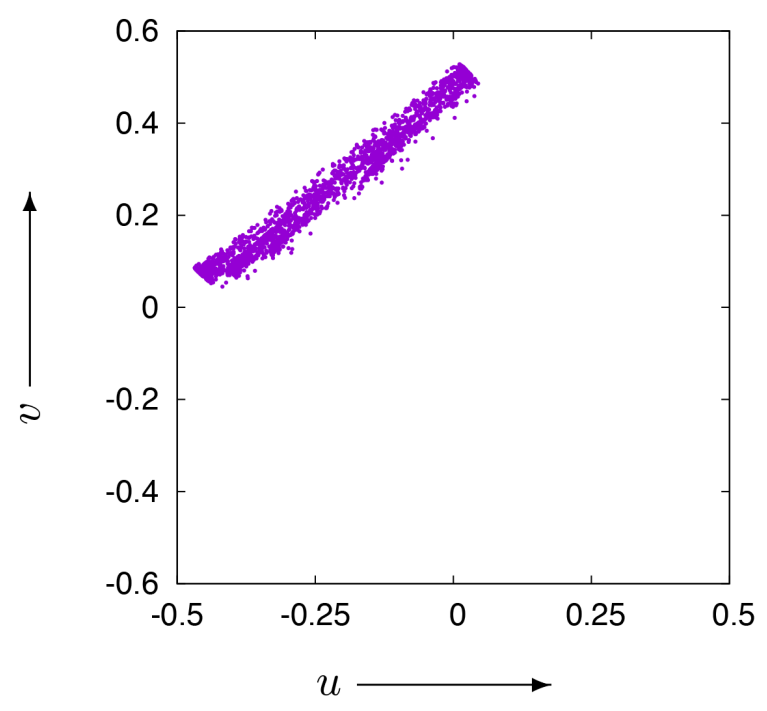

(b) $t=0,1, \ldots, 2600$

Fig. 13. Phase portraits of chaotic itinerancy at $\alpha=12, \beta=-0.504$, and $\varepsilon=-0.086$. 


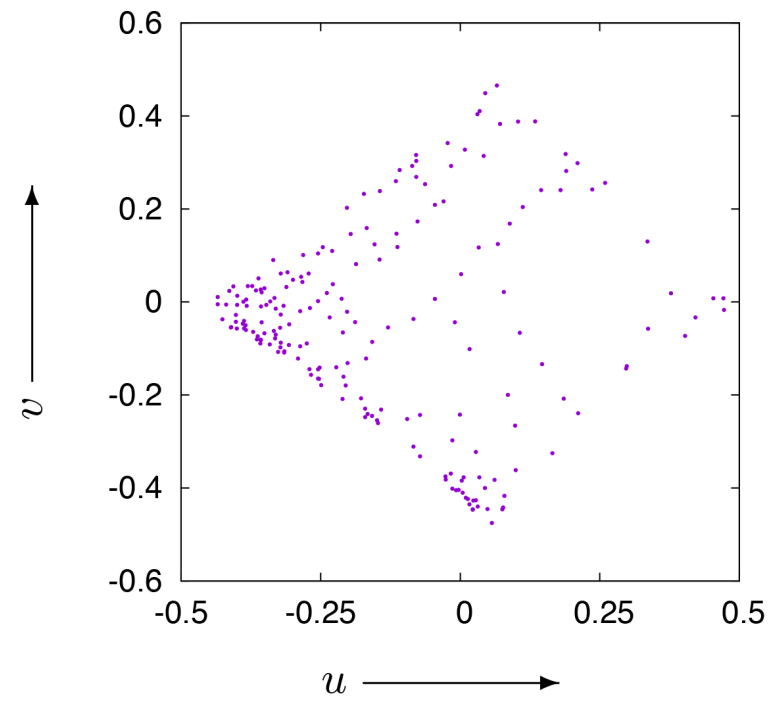

(c) $t=2700,2701, \ldots, 2900$

Fig. 13. (Continued)

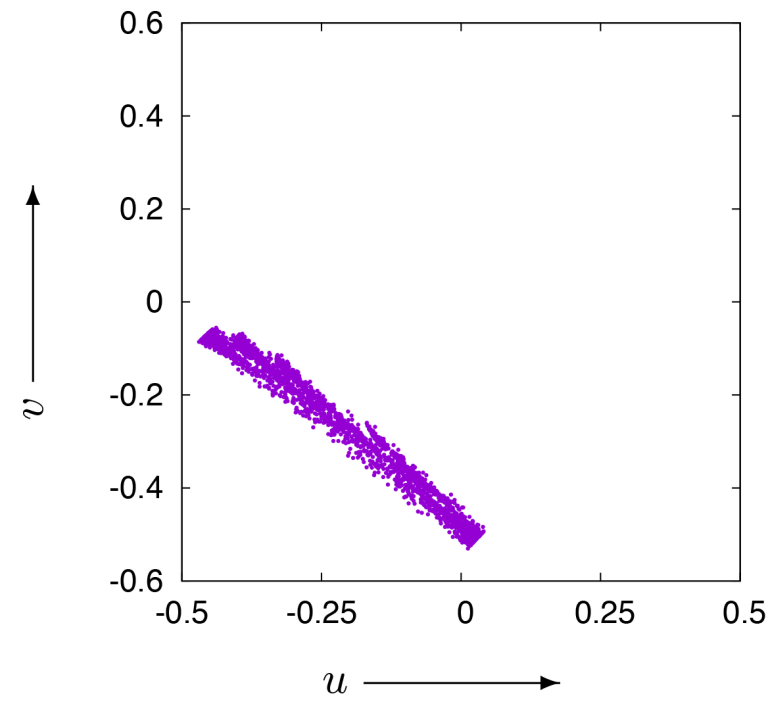

(d) $t=10000,10001, \ldots, 12600$

Fig. 16, the bold solid and dashed curves indicate the stable and unstable fixed points, respectively. The solid curves connected to $\bigcirc$, which signifies $D$-type branching, represents the symmetric stable fixed point. On the contrary, the curves ending at and $\bigcirc$, which indicate the tangent and period-doubling bifurcations, respectively, denote the asymmetric stable fixed point.

The bifurcation structure of the mutually coupled Gaussian map depends on the characteristic of

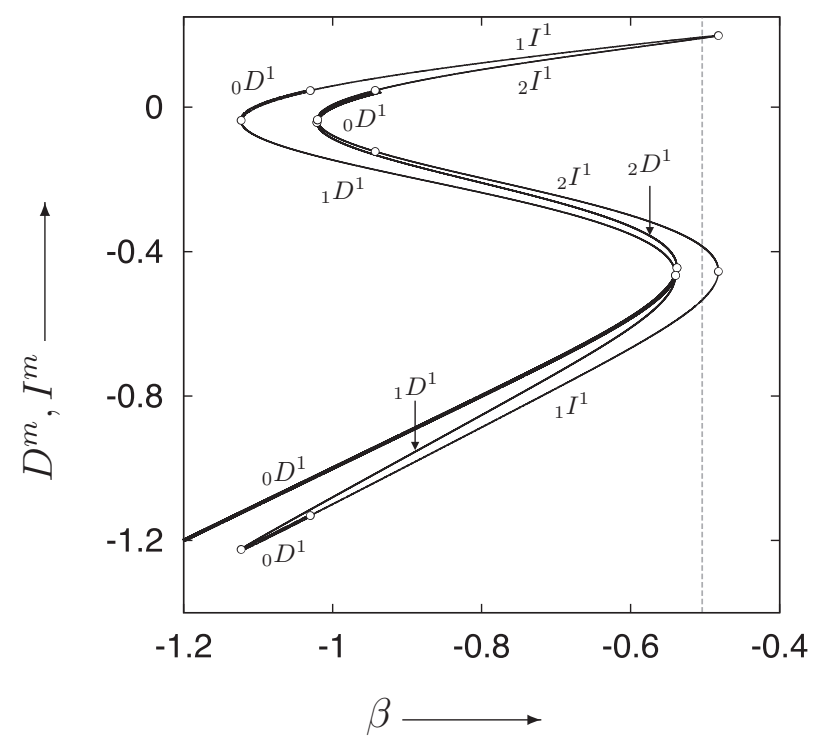

Fig. 15. Stability change of the fixed point at $\varepsilon=-0.086$ and $\alpha=12$. The dashed line indicates $\beta=-0.504$, which corresponds to the parameter set at the X mark in Fig. 14 


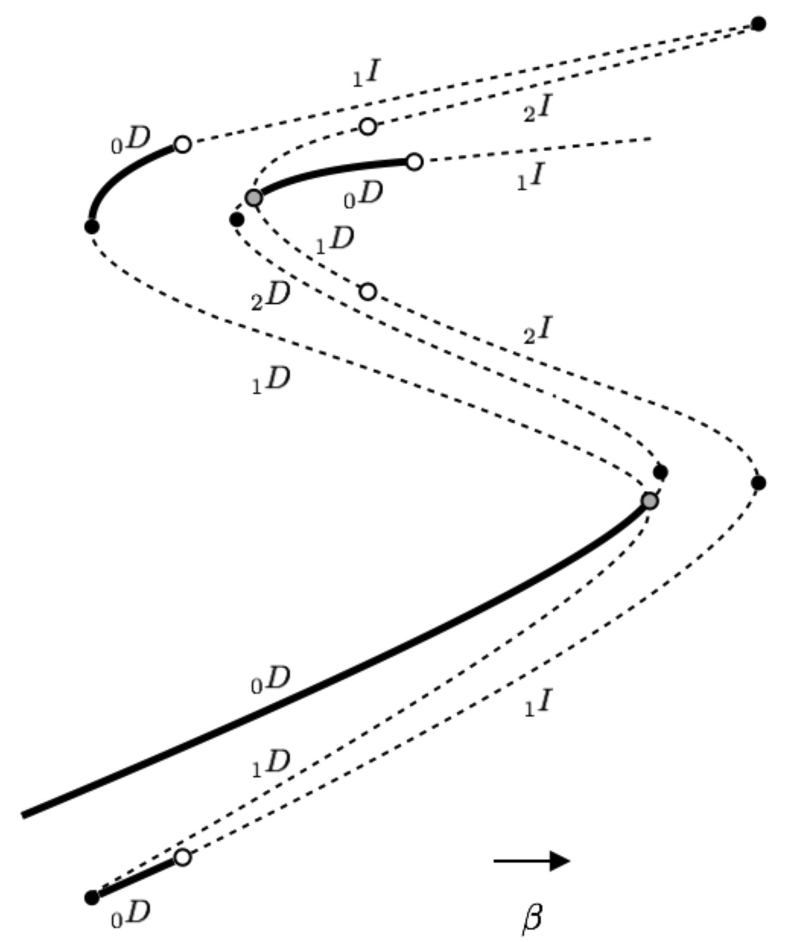

Fig. 16. Schematic of the relation between two $D$-type branchings of the symmetric and asymmetric fixed points.

the single Gaussian map. The range of the function of the single Gaussian map is $(\beta, 1+\beta]$, whereas that of the single logistic map is $(-\infty, \gamma / 4+\beta]$. Hence, it follows that the periodic points observed in the Gaussian map do not diverge and that the fixed point always exists.

When we first focus on the symmetric stable fixed point at the top in Fig. 16, the generation of the asymmetric stable fixed point can be explained as follows. The stability of the fixed point changes

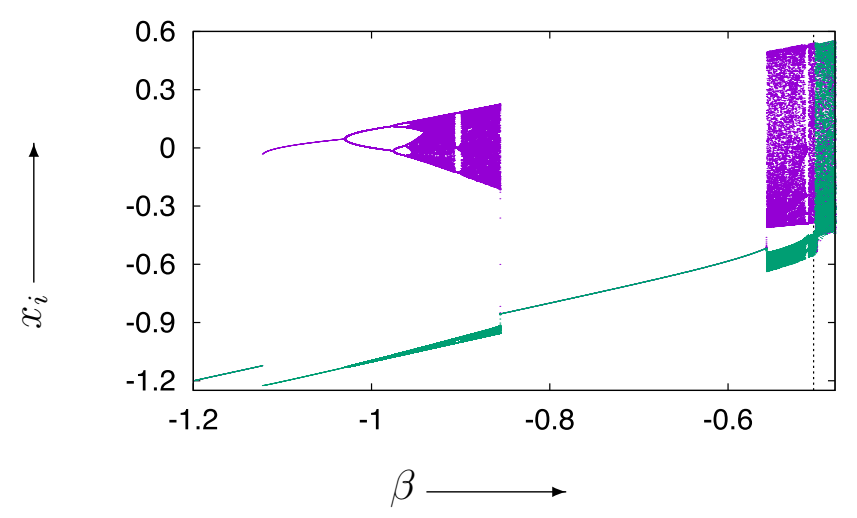

Fig. 17. One-parameter bifurcation diagram observed in the mutually coupled Gaussian map at $\varepsilon=-0.086$ and $\alpha=12$. The dashed line indicates $\beta=-0.504$ which corresponds to the parameter set at the X mark in Fig. 14. to unstable, and the asymmetric unstable fixed point appears around the symmetric stable fixed point. The asymmetric unstable fixed point passes through period-doubling bifurcation with increasing $\beta$ and is terminated with tangent bifurcation. The asymmetric unstable fixed point on the folded manifold passes through the period-doubling bifurcation with decreasing $\beta$, and the stability of the asymmetric fixed point is recovered. Consequently, the asymmetric stable fixed point appears. Interestingly, the asymmetric stable fixed point is also folded by tangent bifurcation at the left-hand end of the parameter region of $\beta$, where the asymmetric stable fixed point exists, and the asymmetric unstable fixed point connects to another symmetric stable fixed point via $D$-type branching. As shown in Fig. [16, the manifold of the asymmetric fixed point has an S-shaped form at $\alpha=12$ and $\varepsilon=-0.086$.

Chaotic itinerancy generation is related to the period-doubling bifurcation of the asymmetric stable fixed point. A cascade of the period-doubling bifurcation from the asymmetric stable fixed point leads to a chaotic set, and chaotic itinerancy appears at $\beta=-0.504$.

Figure 17 shows a one-parameter bifurcation diagram with respect to $\beta$. At approximately $\beta=$ -1.1 , the stable asymmetric fixed point exists. The asymmetric fixed point leads to chaos when $\beta$ is varied. Between $\beta=-0.85$ and -0.58 , the chaotic behavior is not displayed in Fig. 17 because the stable symmetric fixed point coexists in the range of parameter $\beta$. Each chaotic trajectory generated around the asymmetric fixed point becomes closer and ultimately begins to be itinerant at $\beta=-0.504$.

\section{Conclusion}

We investigated the bifurcation structure of the fixed point and periodic points observed in a mutually coupled Gaussian map and a mutually coupled logistic map. In the mutually coupled Gaussian map, complex bifurcation phenomena were observed; however, no such structure existed in the mutually coupled logistic map. Based on the bifurcation analysis, the generation mechanism of the asymmetric stable fixed point was elucidated. In other words, the asymmetric unstable fixed point generated by the $D$-type branching of a symmetric stable fixed point becomes stable by passing through the period-doubling and tangent bifurcations. It is assumed that the stable or unstable fixed 
point always exists with respect to all $\beta$ in the mutually coupled Gaussian map because of the shape of the mapś function. Furthermore, the detailed structure of the change in the stability of the fixed point was clarified based on the bifurcation analysis.

In the mutually coupled Gaussian map, even though it is a two-dimensional discrete-time dynamical system, chaotic itinerancy was observed. The mechanism of the generation of the phenomena was clearly elucidated based on the bifurcation structure of the asymmetric fixed point. We also compared the bifurcation structure of the mutually coupled Gaussian map to that of the mutually coupled logistic map by focusing on $D$-type branching and discussed how chaotic itinerancy in the two-dimensional coupled map only appears in the mutually coupled Gaussian map due to the characteristics of the function.

\section{References}

Aftab, M. S. \& Shafiq, M. [2015] "Neural networks for tracking of unknown SISO discrete-time nonlinear dynamic systems," ISA Trans. 59, 363-374.

Balanov, A. G., Janson, N. B. \& Schöll, E. [2005] "Delayed feedback control of chaos: Bifurcation analysis," Phys. Rev. E 71, 016222.

Banerjee, S. \& Grebogi, C. [1999] "Border collision bifurcations in two-dimensional piecewise smooth maps," Phys. Rev. E 59, 4052-4061.

Berger, C. M., Zhao, X., Schaeffer, D. G., Dobrovolny, H. M., Krassowska, W. \& Gauthier, D. J. [2007] "Period-doubling bifurcation to alternans in paced cardiac tissue: Crossover from smooth to bordercollision characteristics," Phys. Rev. Lett. 99, 058101.

Cho, H., Baum, J. \& Pines, A. [1987] "Iterative maps with multiple fixed points for excitation of two level systems," J. Chem. Phys. 86, 3089-3106.

Gámez, M., López, I., Rodríguez, C., Varga, Z. \& Garay, J. [2017] "Ecological monitoring in a discrete-time prey-predator model," J. Theor. Biol. 429, 52-60.

Gandomi, A. H., Yang, X.-S., Talatahari, S. \& Alavi, A. H. [2013] "Firefly algorithm with chaos," Commun. Nonlin. Sci. Numer. Simul. 18, 89-98.

Gilad, E., von Hardenberg, J., Provenzale, A., Shachak, M. \& Meron, E. [2004] "Ecosystem engineers: From pattern formation to habitat creation," Phys. Rev. Lett. 93, 098105-1-4.

Guckenheimer, J. \& Holmes, P. J. [2013] Nonlinear Oscillations, Dynamical Systems, and Bifurcations of Vector Fields, Vol. 42 (Springer Science \& Business Media).
Han, L., Wang, D. Z. W., Lo, H. K., Zhu, C. \& Cai, X. [2017] "Discrete-time day-to-day dynamic congestion pricing scheme considering multiple equilibria," Transp. Res. B Methodol. 104, 1-16.

Hilborn, R. C. [2000] Chaos and Nonlinear Dynamics (Oxford University Press).

Ikeda, K., Otsuka, K. \& Matsumoto, K. [1989] "Maxwell-Bloch turbulence," Prog. Theor. Phys. Suppl. 99, 295-324.

Kaneko, K. [1991] "Globally coupled circle maps," Physica D 54, 5-19.

Kaneko, K. \& Tsuda, I. [2003] "Chaotic itinerancy," Chaos 13, 926-936.

Kawakami, H. [1984] "Bifurcation of periodic responses in forced dynamic nonlinear circuits: Computation of bifurcation values of the system parameters," IEEE Trans. Circuits Syst. 31, 248-260.

Lampart, M. \& Oprocha, P. [2016] "Chaotic subdynamics in coupled logistic maps," Physica D 335, $45-53$.

Maranhao, M. D. [2016] "Ordered and isomorphic mapping of periodic structures in the parametrically forced logistic map," Phys. Lett. A 380, 3238-3243.

May, R. M. [1976] "Simple mathematical models with very complicated dynamics," Nature 261, 459-467.

Orosz, G., Wilson, R. E. \& Krauskopf, B. [2004] "Global bifurcation investigation of an optimal velocity traffic model with driver reaction time," Phys. Rev. E 70, 026207-1-10.

Patidar, V. [2006] "Co-existence of regular and chaotic motions in the Gaussian map," Electron. J. Theor. Phys. 3, 29-40.

Patidar, V. \& Sud, K. [2009] "A comparative study on the co-existing attractors in the Gaussian map and its $q$-deformed version," Commun. Nonlin. Sci. Numer. Simul. 14, 827-838.

Tarasova, V. V. \& Tarasov, V. E. [2017] "Logistic map with memory from economic model," Chaos Solit. Fract. 95, 84-95.

Tsuda, I. \& Umemura, T. [2003] "Chaotic itinerancy generated by coupling of Milnor attractors," Chaos 13, 937-946.

Tsuda, I. [2015] "Chaotic itinerancy and its roles in cognitive neurodynamics," Curr. Opin. Neurobiol. 31, $67-71$.

Verhulst, P. F. [1845] "Mathematical researches into the law of population growth increase," Nouveaux Mémoires de l'Académie Royale des Sciences et Belles-Lettres de Bruxelles 18, 1-42.

Wieczorek, S., Simpson, T. B., Krauskopf, B. \& Lenstra, D. [2002] "Global quantitative predictions of complex laser dynamics," Phys. Rev. E 65, 045207-1-4. 